\title{
Whole-Brain Diffusion Tensor Imaging in Correlation to Visual-Evoked Potentials in Multiple Sclerosis: A Tract-Based Spatial Statistics Analysis
}

\author{
D. Lobsien, B. Ettrich, K. Sotiriou, J. Classen, F. Then Bergh, and K.-T. Hoffmann
}

\begin{abstract}
BACKGROUND AND PURPOSE: Functional correlates of microstructural damage of the brain affected by MS are incompletely understood. The purpose of this study was to evaluate correlations of visual-evoked potentials with microstructural brain changes as determined by DTI in patients with demyelinating central nervous disease.
\end{abstract}

MATERIALS AND METHODS: Sixty-one patients with clinically isolated syndrome or MS were prospectively recruited. The mean P100 visual-evoked potential latencies of the right and left eyes of each patient were calculated and used for the analysis. For DTI acquisition, a single-shot echo-planar imaging pulse sequence with 80 diffusion directions was performed at 3T. Fractional anisotropy, radial diffusivity, and axial diffusivity were calculated and correlated with mean P100 visual-evoked potentials by tract-based spatial statistics.

RESULTS: Significant negative correlations between mean P100 visual-evoked potentials and fractional anisotropy and significant positive correlations between mean P100 visual-evoked potentials and radial diffusivity were found widespread over the whole brain. The highest significance was found in the optic radiation, frontoparietal white matter, and corpus callosum. Significant positive correlations between mean P100 visual-evoked potentials and axial diffusivity were less widespread, notably sparing the optic radiation.

CONCLUSIONS: Microstructural changes of the whole brain correlated significantly with mean P100 visual-evoked potentials. The distribution of the correlations showed clear differences among axial diffusivity, fractional anisotropy, and radial diffusivity, notably in the optic radiation. This finding suggests a stronger correlation of mean P100 visual-evoked potentials to demyelination than to axonal damage.

ABBREVIATIONS: $A D=$ axial diffusivity; FA = fractional anisotropy; FMRIB = Functional MR Imaging of the Brain; $m$ VEP = mean P100 visual-evoked potentials latencies; RD = radial diffusivity; VEP = visual-evoked potentials

M $\mathrm{R}$ imaging plays a pivotal role in establishing the diagnosis and follow-up of MS. ${ }^{1,2}$ Besides conventional MR imaging, an advanced technique frequently used in MS studies is DTI. ${ }^{3}$ By measuring the diffusion directions of water molecules, DTI is capable of detecting microstructural changes not visible on standard MR imaging. Common parameters obtained from DTI to assess these

Received January 14, 2014; accepted after revision May 16.

From the Departments of Neuroradiology (D.L., K.-T.H., K.S.) and Neurology (B.E., F.T.B., K.S., J.C.), University and University Hospital Leipzig, Leipzig, Germany.

D. Lobsien and B. Ettrich contributed equally to this work.

F. Then Bergh and K.-T. Hoffman contributed equally to this work.

This work was supported by the German Federal Ministry of Education and Research (PtJ-Bio, 0313909, to B.E. and F.T.B.) and the Hellenic Society of Neurology (to K.S.).

Paper previously presented in part as a poster at: Annual Meeting of the American Academy of Neurology, April 21-28, 2012; New Orleans, Louisiana.

Please address correspondence to Donald Lobsien, MD, Department of Neuroradiology, University and University Hospital Leipzig, Liebigstr 20, 04103 Leipzig,

Germany; e-mail: Donald.Lobsien@medizin.uni-leipzig.de

- Indicates open access to non-subscribers at www.ajnr.org

http://dx.doi.org/10.3174/ajnr.A4034 changes are fractional anisotropy (FA) and mean diffusivity. Recently, mean diffusivity was further analyzed and divided into radial diffusivity $(\mathrm{RD})$ and axial diffusivity $(\mathrm{AD})$, parameters that allow a more specific approach to the underlying microstructural changes. ${ }^{4,5}$ $\mathrm{AD}$ was shown to be the primary correlate for histologic markers of axonal integrity, ${ }^{4}$ while $\mathrm{RD}$ was suggested to provide a specific tool to assess demyelination, distinct from axonal damage. ${ }^{5}$

Pattern reversal visual-evoked potentials (VEP) are an established tool for the evaluation of the optic pathway within the diagnostic work-up of MS, especially as a marker of the integrity of the prechiasmal part. ${ }^{6-8}$ However, they failed to provide reliable results in predicting lesions of the retrochiasmal part. ${ }^{9,10}$ Evoked potentials, in general, and combined evoked potential analysis, in particular, seem to correlate well with the long-term disability of patients with MS. Correlations of evoked potentials and MR imaging are reported controversially. While some studies showed no correlations between evoked potentials and MR imaging, ${ }^{11}$ other studies reported significant correlations between VEP and T2 lesion load. ${ }^{12}$ However, to the best of our knowledge, a correlation of DTI with VEP has not been published. 


\section{Patient characteristics}

\begin{tabular}{lc}
\hline \multicolumn{2}{c}{ Characteristics } \\
\hline Patients (No.) & 61 (41 women, 20 men) \\
Age (yr) & $38.4 \pm 12.04$ \\
Diagnosis: & \\
$\quad$ RR-MS & 49 \\
CIS & 10 \\
PP-MS & 1 \\
$\quad$ Recurrent optic neuritis & 1 \\
Total patients with optic neuritis (No.) & 23 (2.7 $\pm 1.3 \mathrm{yr})$ \\
$\quad$ & \\
$\quad$ (duration before inclusion in the & \\
Mean EDSS score & $2.46 \pm 1.95$ \\
Mean visual acuity & $0.85 \pm 0.24$ \\
mVEP (ms) & $113.05 \pm 14.26$ \\
\hline
\end{tabular}

Note:-CIS indicates clinically isolated syndrome; RR-MS, relapsing-remitting MS; PP-MS, primary-progressive MS; EDSS, Expanded Disability Status Scale.

The aim of our study was, therefore, to evaluate the correlation of VEP with parameters derived from DTI with the following objectives: 1) to identify potential relations of VEP to microstructural changes, and 2) to analyze whether such changes reflect demyelination or axonal damage.

\section{MATERIALS AND METHODS}

The study was approved by the local ethics committee of the University of Leipzig.

\section{Patients}

Sixty-one patients (41 women, 20 men) with clinically isolated syndrome or MS were prospectively recruited from the Neuroimmunology Clinic of the Department of Neurology. Ten patients were classified as having clinically isolated syndrome, 49 had definite MS with a relapsing-remitting course, 1 had primary-progressive MS, and 1 had recurrent optic neuritis but did not fulfill the criteria for neuromyelitis optica. The mean age was $38.4 \pm$ 12.0 years, and the mean Expanded Disability Status Scale score was $2.46 \pm 1.95$. All patients underwent a neurologic examination, a standard VEP examination, and brain MR imaging, including DTI at 3T (see below). Clinical data are shown in the Table. All clinical, electrophysiologic, and imaging examinations in each patient were performed within 3 months. Patients were free of an acute relapse as defined by clinical criteria (history and neurologic examination) for at least 6 months before the first examination and during the study period. None of the patients had a gadolinium-enhancing lesion on T1-weighted images after contrast administration.

\section{Visual-Evoked Potentials}

All VEP were recorded by using the same electrophysiologic device (Medelec Synergy; Oxford Instruments, Oxfordshire, United Kingdom). Stimulation was performed according to standard protocols by checkerboard pattern reversal by using a computer screen at a distance of $1.25 \mathrm{~m}$ with a check size of 60 minutes of arc. Latencies of N70 and P100 and N70/P100 amplitudes were determined for all recordings with identifiable VEP. Normal mean laboratory values for P100 latency were determined previously in 30 healthy, 20- to 60-year-old controls at $101.0 \pm 6.0 \mathrm{~ms}$. The mean P100 VEP latencies (mVEP) of the right and the left eyes of each patient were calculated.

\section{MR Imaging}

DTI scans were acquired on a clinical 3T MR imaging scanner (Magnetom Trio; Siemens, Erlangen, Germany). A single-shot, echo-planar imaging pulse sequence with the following parameters was performed in all patients: TR, $2700 \mathrm{~ms}$; TE, $93 \mathrm{~ms}$; flip angle, $90^{\circ}$; parallel imaging; 80 diffusion-encoding gradient directions; 1 B0 image; $b=1000 \mathrm{~s} / \mathrm{mm}^{2}$; matrix size, $128 \times 128$; voxel size, $1.8 \times 1.8 \times 5 \mathrm{~mm}$. Additionally, standard diagnostic MR imaging sequences were applied, including FLAIR and a T1weighted axial sequence, after the administration of gadolinium contrast at a standard dosage. A 12-channel head coil was used for all sequences.

\section{DTI Data Analysis}

DTI analysis was performed in a standardized, voxel-based, raterindependent manner by applying tract-based spatial statistics, which belongs to the Functional MRI of the Brain Software Library (FMRIB [FSL; http://www.fmrib.ox.ac.uk/fsl]). ${ }^{13}$

Images were preprocessed by using eddy_correct, the Brain Extraction Tool, and DTIFit in FSL to correct motion and eddy current artifacts, extract the brain, and calculate $\mathrm{FA}, \mathrm{AD}$, and $\mathrm{RD}$. All subjects' FA data were then aligned to a $1 \times 1 \times 1 \mathrm{~mm}$ standard space (FMRIB58_FA) and averaged, and the mean FA image was skeletonized. The skeleton was then thresholded at an FA value of 0.2. Following this step, each FA image was projected onto the mean FA skeleton common to all subjects. The same nonlinear transformations derived from the FA maps were applied to the $\mathrm{AD}$ and $\mathrm{RD}$ maps.

The imaging data were subsequently analyzed by using the Randomise Tool (http://www.fmrib.ox.ac.uk/fsl/randomise/). We set up the General Linear Model design with mVEP as a continuous variable of interest and tested for correlations with diffusion parameters (FA, RD, AD), controlled for the effect of age and sex (covariates of noninterest). All the data were mean-centered. Cluster-like structures were enhanced by using the threshold-free cluster enhancement method. Statistics were built over 10,000 permutations with the maximum threshold-free cluster enhancement recorded at each permutation. The 95th percentile of this distribution was used as a threshold-free cluster enhancement threshold, and the significance level was calculated from this distribution. Thus, significant clusters were fully corrected for family-wise error at $P<.05$.

Additionally, we calculated the mean $\mathrm{FA}, \mathrm{RD}$, and $\mathrm{AD}$ in 2 manually selected ROIs of the skeleton. These ROIs correspond to the thalamic radiations (including the optic radiations) according to the ICBM DTI-81 Atlas (http://www.loni.usc.edu/ICBM/ Downloads/Downloads_DTI-81.shtml), which is included in the FMRIB Software Library package. The results of each subject (1 value per patient and parameter, ie, the mean of the 2 ROIs) and each DTI-derived parameter were correlated with the mVEP by using the Spearman rank coefficient. The correlation was performed with GraphPad Prism 4 software (GraphPad Software, San Diego, California).

\section{Lesion Maps}

The T2-FLAIR datasets of all patients were coregistered to a $1 \times$ $1 \times 1 \mathrm{~mm}$ standard space (Montreal Neurological Institute 156)

AJNR Am J Neuroradiol 35:2076-81 Nov 2014 www.ajnr.org 


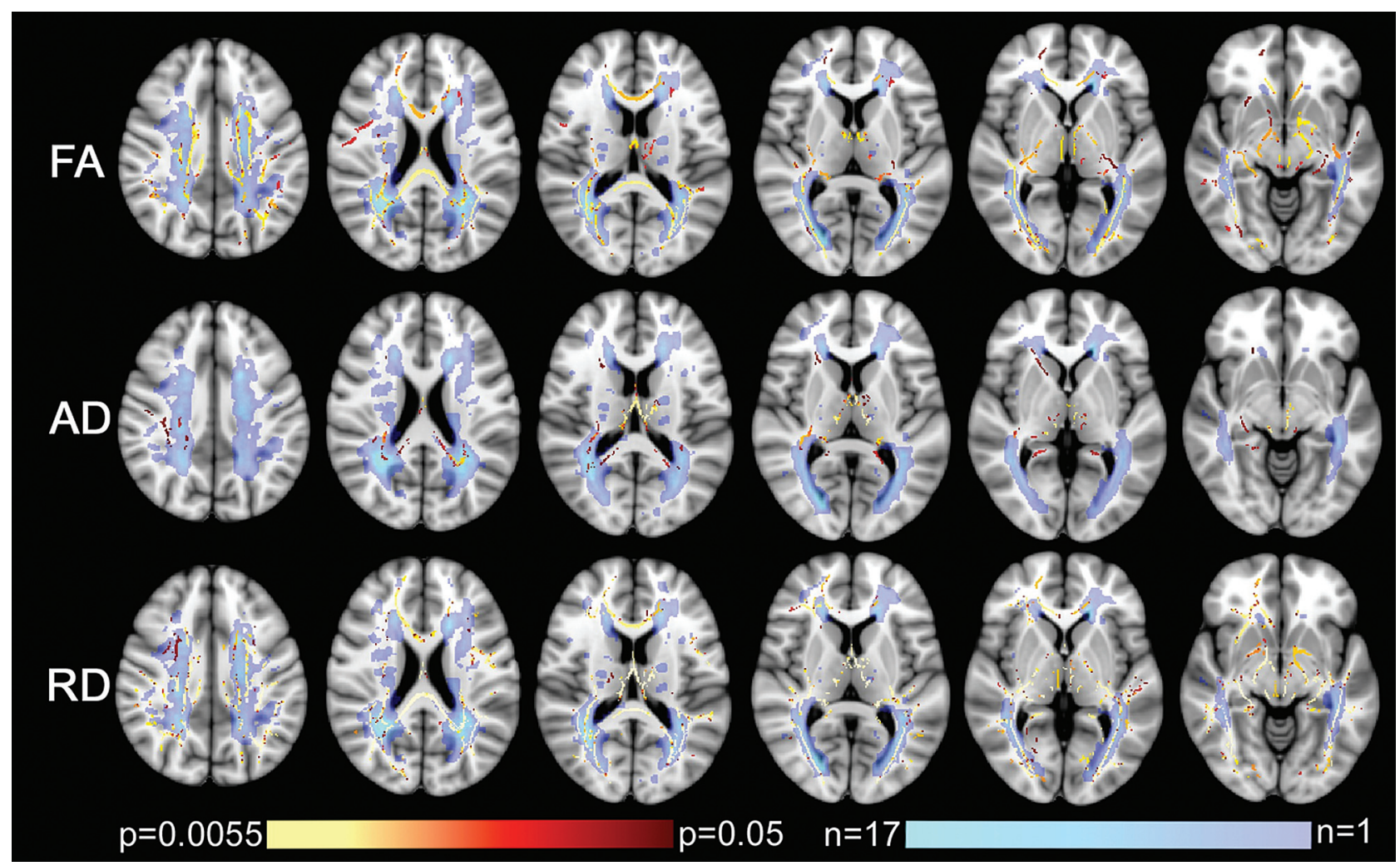

FIG 1. Correlations among FA, RD, AD, and mVEP. Shown are the tract-based spatial statistics results of the correlation analysis and the MS lesion distribution. Significant correlations are shown in red to yellow, from $P=.05$ to $P=.0055$. The MS lesion maps (a summation of all patients) are presented in violet to turquoise. Violet indicates an area where only 1 patient had a lesion, while turquoise indicates an area where up to 17 patients had a lesion. The background image is the T1 image of the Montreal Neurological Institute 158 brain. First line (FA): correlation of FA and mVEP. Second line (AD): correlation of AD and mVEP. Third line (RD): correlation of RD and mVEP. Correlations with FA and RD are present in widespread areas, while there are fewer correlations with AD. Highly significant correlations can be identified in the optic radiations among mVEP, FA, and RD, while no correlation could be found between mVEP and AD. Conversely, there are highly significant correlations between $\mathrm{mVEP}$ and all measured parameters of DTI within the fornix. T2 lesions are predominantly found along the ventricles. The parieto-occipital region is the location where most patients had lesions.

by using the FMRIB Linear Image Registration Tool to assess white matter lesions. Hyperintense lesions on the T2-FLAIR images of all patients were manually segmented and transformed into binary masks by using FSLView (http://fsl.fmrib.ox.ac.uk/fsl/ fslview/). Afterward, the masks were summarized with FMRIB's fslmaths and plotted by using a colorized scale to indicate regions with a high or low incidence of lesions among the subjects. The resulting maps were merged with the results of the tract-based spatial statistics analysis to qualitatively identify whether changes were related to normal-appearing white matter or to lesions.

\section{RESULTS}

\section{Whole-Brain Fractional Anisotropy}

Significant negative correlations were found between FA and mVEP (the longer the mVEP, the more decreased was the FA) in widespread regions of the brain. The most significant regions (up to $P<.0055)$ were located in the optic radiation, frontoparietal white matter, medial thalamus, fornix bilaterally, and splenium of the corpus callosum. The correlations within the splenium of the corpus callosum and in the fornix included areas of predominantly normal-appearing white matter. Further significant correlations $(P<.05)$ were found in multiple locations along the skeleton, including the genu of the corpus callosum, the frontoparietal white matter, and the internal capsule bilaterally. In the genu of the corpus callosum and the adjacent frontal white matter, a slight asymmetry was detected with a more significant correlation on the right side, while, in the internal capsule, the correlations were a little more significant on the left side. Results are shown in Fig 1.

\section{Whole-Brain Radial Diffusivity}

Multiple significant correlations were found between RD and $\mathrm{mVEP}$ (the longer the mVEP the more increased was the RD) along the skeleton. Correlations with the highest significance (up to $P<.0055$ ) were found in the corpus callosum and bilaterally in the frontoparietal white matter, thalamus, fornix, internal capsule, and optic radiation. In the corpus callosum and the right frontal white matter and the left frontotemporal white matter, these correlations were located in normal-appearing white matter. Voxels of lower significance could be found as well in all these areas. There was a slight asymmetry with more significant correlations in the right frontal white matter. Results are shown in Fig 1.

\section{Whole-Brain Axial Diffusivity}

Compared with the widespread regions in which FA and RD correlated to $\mathrm{mVEP}$, much more restricted areas showed a positive correlation between AD and mVEP. Correlations with the highest 


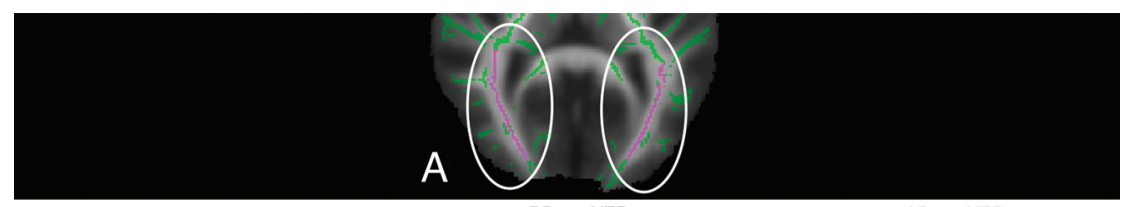

FA vs. VEP

RD vs. VEP

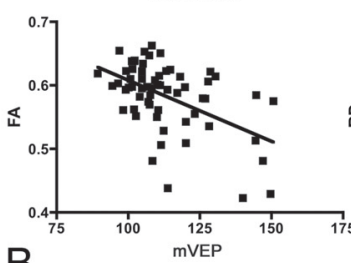

$\mathrm{B}$
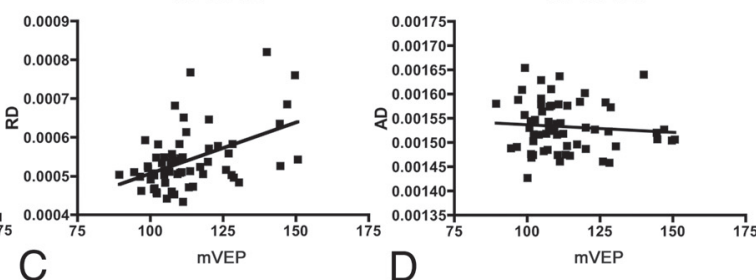

FIG 2. Correlations among mean FA, RD, and AD with mVEP in the optic radiation. $A$, The 2 manually selected ROls of the skeleton corresponding to thalamic radiations (including the optic radiations) according to the ICBM DTI-81 Atlas in the FMRIB Software Library package are shown in purple on the green skeleton. The results of each subject (1 value per patient and parameter, ie, the mean of the 2 ROIs) and each DTI-derived parameter were correlated with the mVEP. $B$, The highly significant negative correlation of the mVEP with the mean FA derived from these particular ROls is shown (Spearman $r=-0.432, P$ [2-tailed] $=.0005$ ). $C$, The highly significant positive correlation of the mean VEP with the mean RD measured in the ROls is shown (Spearman $r=0.398, P$ [2-tailed] $=.0015) . D$, The missing correlation of the mean AD derived from the ROIs and the mean VEP is shown (Spearman $r=-0.097, P$ [2-tailed] $=.458$ ). areas of the brain regarding almost the whole skeleton, while Onu et $\mathrm{al}^{16}$ found significant correlations between clinical motor scores and both FA and RD but not AD.

The widespread correlations of mVEP in our study can be considered an in vivo equivalent to the observations in a postmortem study by Klawiter et $\mathrm{al}^{17}{ }^{17}$ in which RD was predominantly associated with demyelination and, to a much lesser degree, with axonal loss; hence, RD was postulated as a marker of overall tissue integrity within MS lesions. AD, conversely, seemed to be a less sensitive marker because it showed a poor correlation with axonal loss in the same study. Furthermore, postmortem studies suggested that $\mathrm{RD}$ and FA could be considered surrogates of cerebral myelin content in patients with MS. ${ }^{18}$ In mice, RD was identified as a demyelination marker, and $\mathrm{AD}$ significance $(P<.0055)$ were found only in the fornix and thalamus bilaterally. Correlations of lower significance were found bilaterally in the splenium of the corpus callosum, the periventricular parietal white matter, the frontoparietal white matter, the right frontal white matter, and the anterior limb of the right internal capsule. There were no correlations in the optic radiations. Results are shown in Fig 1.

\section{Optic Radiation Region-of-Interest Analysis}

There was a highly significant correlation in the ROIs along the skeleton corresponding to the optic radiation between mean RD and mVEP, a highly negative correlation between mean FA and mVEP, and no correlation between mean AD and mVEP. The results are shown in Fig 2.

\section{DISCUSSION}

We analyzed the relationship between VEP latencies and cerebral microstructure as measured by different parameters of DTI in 61 subjects with demyelinating diseases. A strong positive correlation was found between $\mathrm{MVEP}$ and $\mathrm{RD}$, and a strong negative correlation, between $\mathrm{mVEP}$ and FA in various areas of the brain, whereas very few positive correlations were found between $\mathrm{AD}$ and $\mathrm{mVEP}$.

\section{Analysis of Whole-Brain Correlation Patterns}

We found far more correlations of the mVEP with RD and FA than with $\mathrm{AD}$. Similar patterns of $\mathrm{RD}, \mathrm{AD}$, and FA were reported previously, despite different study designs or correlations with different clinical parameters.

For example, in a study by Liu et al, ${ }^{14}$ the degree of disability of patients with MS correlated strongly with RD and FA but not with $\mathrm{AD}$ in disseminated areas of the brain, including the thalamus and optic radiation. Additionally, they found correlations among RD, FA, and disease duration, which, in turn, correlated poorly with AD. Sbardella et $\mathrm{al}^{15}$ reported a strong correlation between multiple sclerosis functional composite subscales and FA in various was identified as an axonal damage marker. ${ }^{5}$

In accordance with the literature, our results seem to support the hypothesis that RD could possibly serve as an overall marker for tissue damage caused by MS that correlates not only with clinical parameters of cognitive and motor impairment but also with results of electrophysiologic examinations of the visual system. The prolonged VEP latency might be caused mainly by demyelination, which seems reasonable, because myelination is a main determinant in the speed of action-potential propagation along the axon.

\section{Analysis of the Visual Tract}

We found a strong correlation between mVEP and RD as well as $\mathrm{FA}$, but not with $\mathrm{AD}$, when focusing on the optic radiation, similar to whole-brain analysis. In the literature, similar patterns of $\mathrm{RD}, \mathrm{AD}$, and FA have been reported, despite different study designs and correlations with different clinical parameters.

Liu et $\mathrm{al}^{19}$ found significant differences between patients and controls in the optic radiation that were present bilaterally in $\mathrm{RD}$ and mean diffusivity but only unilaterally in the AD analysis. In another study, they also found significant differences in the optic radiation between patients and controls for $\mathrm{RD}$ and $\mathrm{FA}$ but not for AD. ${ }^{14}$ Rueda Lopes et $\mathrm{al}^{20}$ also found significant differences within the optic radiation for $\mathrm{RD}$ and FA but not for $\mathrm{AD}$ in their study of patients with neuromyelitis optica. Roosendaal et $\mathrm{al}^{21}$ found a significantly lower FA within the optic radiation, including areas with MS lesions and normal-appearing white matter in patients with MS in comparison with healthy controls. Onu et $\mathrm{al}^{16}$ found a significant difference between patients with MS and controls for RD and FA in the optic radiation. However, in a recent study, Kolbe et $\mathrm{al}^{22}$ found no correlation of visual acuity in optic neuritis with DTI parameters within the optic radiation but did find a correlation within prefrontal and temporal brain regions.

Optic pathway involvement is common in MS, in the acute phase and in chronic stages. ${ }^{23}$ VEP show a correlation with measures of visual acuity and disease severity, though there are con-

AJNR Am J Neuroradiol 35:2076-81 Nov 2014 www.ajnr.org 
troversies regarding the correlation with the clinical course of the disease. ${ }^{8}$ If we take these aspects into account, the relation between the electrophysiologic function (mVEP) and the microstructural damage (DTI) we found seems very reasonable.

\section{Analysis of Normal-Appearing White Matter and Deep Gray Matter of the Fornix and Thalamus}

In contrast to most other areas that showed correlations to mVEP, we found a highly significant correlation of mVEP with both an $\mathrm{AD}$ and $\mathrm{RD}$ increase and with an FA decrease in the fornix bilaterally and in the medial aspects of the thalamus, all being areas of normal-appearing white or deep gray matter, respectively.

Concerning changes of the fornix in MS, the literature is limited. Kern et $\mathrm{al}^{24}$ found significantly reduced FA in patients with MS compared with healthy controls in the fornix in a study of verbal memory function. Higher FA values correlated with a better performance in verbal memory tests in patients. The authors concluded that successful functional compensation of hippocampal involvement might be limited in part by damage to the fornix, consistent with the critical role of this pathway in the clinical expression of memory impairment in MS. This limitation could be a direct consequence of demyelination and an indirect result of primary hippocampal damage. ${ }^{25}$

Roosendaal et $\mathrm{al}^{21}$ found clearly reduced FA in both fornices by using a tract-based spatial statistics-based analysis, mainly focusing on normal-appearing white matter. Similar to Kern et al, ${ }^{24}$ they assumed at least 2 reasons for this: 1) secondary degeneration caused by disseminated focal demyelination of the hippocampus, and 2) direct demyelination of the fornix, both of which had been demonstrated in postmortem studies of patients with MS. Experimental studies in rats identified important functions of hippocampal projections for spatial-visual perception, and animals with fornix lesions performed substantially worse than healthy ones in visual shape recognition. ${ }^{25,26}$

It is unclear why in our study, mVEP correlated so significantly with all 3 DTI metrics in this specific white matter tract that is not directly associated with visual function. A hypothesis is that secondary and primary pathologic changes might play a role in this structure; therefore, both demyelination and axonal damage are present. This hypothesis could explain the significant correlation with $\mathrm{AD}, \mathrm{RD}$, and $\mathrm{FA}$ in the fornix in contrast to the correlation patterns in most other brain structures.

Regarding the thalamus in MS, there is very little literature dealing specifically with DTI. Extensive, confluent, subependymal thalamic demyelination is relatively common as is diffuse microglial activation in normal-appearing thalami, indicating direct and secondary tissue damage. ${ }^{27}$ This finding seems to support ours of rather mixed changes with a correlation pattern pointing to various causes of thalamic tissue alterations in demyelinating disease (ie, both primarily and secondarily induced damage).

\section{Limitations}

There are some limitations of our study. The patient sample was not homogeneous in terms of sex. Although reflecting the normal sex distribution of MS, an implication on the results is possible because sex-specific differences in various aspects of DTI analyses were found in other studies. ${ }^{28}$ Therefore, we corrected for sex as a covariate of noninterest. However, because our major aim was to analyze the correlation between mVEP and DTI and not to evaluate sex differences, we did not perform subgroup analyses.

Concerning technical aspects, the DTI sequence used has a relatively low resolution on the z-axis. This could bias the detection of correlations, especially in small structures, to an unknown extent. However, the technique used is in line with recent literature referring to this topic, which is relatively heterogeneous. For example, in the literature, section thickness ranges from approximately 2-5 mm; some studies use gaps between sections and varying field strengths, and various numbers of diffusion gradients (among other parameters) are applied. ${ }^{16,19,21}$ Finally, the exact histopathologic processes responsible for changes detected by DTI are understood only in part; therefore, conclusions must be interpreted with caution.

\section{CONCLUSIONS}

Our results show that widespread microstructural changes of the whole brain in MS correlate significantly with VEP. Clear differences among $\mathrm{AD}, \mathrm{FA}$, and $\mathrm{RD}$, notably in the optic radiation, suggest a stronger correlation of $\mathrm{mVEP}$ with demyelination than with axonal damage. In conjunction with the literature, our results support the hypothesis that the parameters FA, RD, and $\mathrm{AD}$ could serve potentially as relevant imaging markers for demyelinating disease staging and burden quantification beyond the application in clinical studies.

\section{ACKNOWLEDGMENTS}

We thank Alfred Anwander, MD, for helpful technical hints with DTI data analysis.

Disclosures: Barbara Ettrich—RELATED: Grant: Federal Ministry of Education and Research, through the Translational Centre for Regenerative Medicine.* Konstantinos Sotiriou—RELATED: Grant: Hellenic Neurological Society, Comments: I received a grant from the Hellenic (Greek) Neurological society, for my fellowship in the Neuroradiology Department of the University Hospital in Leipzig, Germany. Florian Then BerghRELATED: Grant: Federal Ministry of Education and Research through the Translational Centre for Regenerative Medicine, ${ }^{*}$ UNRELATED: Board Membership: I received personal compensation for activities with Bayer, Biogen Idec, Novartis, Merck-Serono, and Sanofi-Aventis Pharmaceuticals as a speaker and/or member on an advisory board, Grants/Grants Pending: I received research support from Bayer Pharmaceuticals Corporation* and Teva Neuroscience, ${ }^{*}$ Payment for Lectures (including service on Speakers Bureaus): I received personal compensation for activities with Bayer, Biogen Idec, Novartis, Merck-Serono, and Sanofi-Aventis Pharmaceuticals as a speaker and/or member on an advisory board. *Money paid to the institution.

\section{REFERENCES}

1. Ciccarelli O, Toosy AT, Hickman SJ, et al. Optic radiation changes after optic neuritis detected by tractography-based group mapping. Hum Brain Mapp 2005;25:308-16

2. Vigeveno RM, Wiebenga OT, Wattjes MP, et al. Shifting imaging targets in multiple sclerosis: from inflammation to neurodegeneration. J Magn Reson Imaging 2012;36:1-19

3. Filippi M, Rocca MA, De Stefano N, et al. Magnetic resonance techniques in multiple sclerosis: the present and the future. Arch Neurol 2011;68:1514-20

4. Budde MD, Xie M, Cross AH, et al. Axial diffusivity is the primary correlate of axonal injury in the experimental autoimmune encephalomyelitis spinal cord: a quantitative pixelwise analysis. J Neurosci 2009;29:2805-13

5. Song SK, Yoshino J, Le TQ, et al. Demyelination increases radial diffusivity in corpus callosum of mouse brain. Neuroimage 2005;26:132-40 
6. Halliday AM, McDonald WI, Mushin J. Visual evoked response in diagnosis of multiple sclerosis. Br Med J 1973;4:661-64

7. Lascano AM, Brodbeck $\mathrm{V}$, Lalive $\mathrm{PH}$, et al. Increasing the diagnostic value of evoked potentials in multiple sclerosis by quantitative topographic analysis of multichannel recordings. J Clin Neurophysiol 2009;26:316-25

8. Weinstock-Guttman B, Baier M, Stockton R, et al. Pattern reversal visual evoked potentials as a measure of visual pathway pathology in multiple sclerosis. Mult Scler 2003;9:529-34

9. Benbadis SR, Lancman ME, Wolgamuth BR, et al. Value of full-field visual evoked potentials for retrochiasmal lesions. J Clin Neurophysiol 1996;13:507-10

10. Plant GT, Kermode AG, Turano G, et al. Symptomatic retrochiasmal lesions in multiple sclerosis: clinical features, visual evoked potentials, and magnetic resonance imaging. Neurology 1992;42:68-76

11. Schlaeger R, D'Souza M, Schindler C, et al. Combined evoked potentials as markers and predictors of disability in early multiple sclerosis. Clin Neurophysiol 2012;123:406-10

12. Kantorová E, Ziak P, Kurca E, et al. Visual evoked potential and magnetic resonance imaging are more effective markers of multiple sclerosis progression than laser polarimetry with variable corneal compensation. Front Hum Neurosci 2014;8:10

13. Smith SM, Jenkinson M, Johansen-Berg H, et al. Tract-based spatial statistics: voxelwise analysis of multi-subject diffusion data. Neuroimage 2006;31:1487-505

14. Liu Y, Duan Y, He Y, et al. Whole brain white matter changes revealed by multiple diffusion metrics in multiple sclerosis: a TBSS study. Eur J Radiol 2012;81:2826-32

15. Sbardella E, Petsas N, Tona F, et al. Assessing the correlation between grey and white matter damage with motor and cognitive impairment in multiple sclerosis patients. PloS One 2013;8:e63250

16. Onu M, Roceanu A, Sboto-Frankenstein U, et al. Diffusion abnormality maps in demyelinating disease: correlations with clinical scores. Eur J Radiol 2012;81:e386-91

17. Klawiter EC, Schmidt RE, Trinkaus K, et al. Radial diffusivity pre- dicts demyelination in ex vivo multiple sclerosis spinal cords. $\mathrm{Neu}$ roimage 2011;55:1454-60

18. Schmierer K, Wheeler-Kingshott CA, Boulby PA, et al. Diffusion tensor imaging of post mortem multiple sclerosis brain. Neuromage 2007;35:467-77

19. Liu $Y$, Duan $Y, H e Y$, et al. A tract-based diffusion study of cerebral white matter in neuromyelitis optica reveals widespread pathological alterations. Mult Scler 2012;18:1013-21

20. Rueda Lopes FC, Doring T, Martins C, et al. The role of demyelination in neuromyelitis optica damage: diffusion-tensor MR imaging study. Radiology 2012;263:235-42

21. Roosendaal SD, Geurts JJ, Vrenken H, et al. Regional DTI differences in multiple sclerosis patients. Neuroimage 2009;44:1397-403

22. Kolbe SC, Marriott M, Walt Av, et al. Diffusion tensor imaging correlates of visual impairment in multiple sclerosis and chronic optic neuritis. Invest Ophthalmol Vis Sci 2012;53:825-32

23. Jasse L, Vukusic S, Durand-Dubief F, et al. Persistent visual impairment in multiple sclerosis: prevalence, mechanisms and resulting disability. Mult Scler 2013;19:1618-26

24. Kern KC, Ekstrom AD, Suthana NA, et al. Fornix damage limits verbal memory functional compensation in multiple sclerosis. Neuroimage 2012;59:2932-40

25. Gaffan EA, Bannerman DM, Warburton EC, et al. Rats' processing of visual scenes: effects of lesions to fornix, anterior thalamus, mamillary nuclei or the retrohippocampal region. Behav Brain Res 2001;121:103-17

26. Thomas AG, Koumellis P, Dineen RA. The fornix in health and disease: an imaging review. Radiographics 2011;31:1107-21

27. Minagar A, Barnett MH, Benedict RH, et al. The thalamus and multiple sclerosis: modern views on pathologic, imaging, and clinical aspects. Neurology 2013;80:210-19

28. Schoonheim MM, Vigeveno RM, Rueda Lopes FC, et al. Sex-specific extent and severity of white matter damage in multiple sclerosis: implications for cognitive decline. Hum Brain Mapp 2014;35: $2348-58$ 